\title{
5R y Sustentabilidad hospitalaria: Nuestro aporte en la emergencia climática. Parte II. Repensar e investigar
}

\author{
5R and Hospital Sustainability: Our contribution in the climate emergency. \\ Part II. Rethink and research
}

Waldo Merino MSc, MBA. ${ }^{1, *}$, Isabel Quispe PhD, MSc. ${ }^{2}$, Christian Guentelicán ${ }^{3}$, Sebastián Herrero ${ }^{3}$, Robin Estrada ${ }^{4}$, Sebastián Inostroza ${ }^{3}$, Gonzalo Henríquez³ ${ }^{3}$ Claudio Cárcamo ${ }^{5}$, Francisco Rubilar MSc. ${ }^{5}$, Patricia Mogrovejo ${ }^{4}$

1 Departamento de Cirugía, Traumatología y Anestesia. Centro de Capacitación Investigación y Gestión en Salud para la Medicina Basada en Evidencias (CIGES), Facultad de Medicina, Universidad de La Frontera. Temuco, Chile.

2 Red Peruana de Ciclo de Vida y Ecología Industrial, Departamento de Ingeniería, Pontificia Universidad Católica del Perú. Lima, Perú.

3 Instituto del Medio Ambiente Universidad de La Frontera. Temuco, Chile.

4 Interno de Medicina, Universidad de La Frontera. Temuco, Chile.

5 Estudiante de Ingeniería Industrial, Pontificia Universidad Católica del Perú. Lima, Perú.

Financiamiento: Proyecto CF-HEALTHSUD (código 21- CLIMAT-03; Climat Amsud).

Agradecimientos: Se agradece a Fondecyt (Perú) por el soporte brindado.

Declaramos que ninguno de los autores de este trabajo presenta conflictos de interés.

Fecha de recepción: 22 de abril de 2021 / Fecha de aceptación 08 de julio de 2021

\begin{abstract}
Introduction: Climate change is a health problem and, at the same time, health systems are important contributors. Hospitals stand out due to their high rates of energy consumption, resources and waste generation. The purpose of the study is to know and identify the determinants of sanitary waste and the measures that can be implemented that allow reducing the production of hospital waste, seeking to achieve a general and updated appreciation of this phenomenon and taking into account hospital sustainability. Method: A bibliographic search was carried out in pubmed that included keywords related to the concepts of carbon footprint, recycling and hospital waste. The screening yielded a total of 37 articles and later 12 publications founded from references (or that were previously known by the authors) were added. Results: The results are presented into 5 points known as the " 5 Rs", named below. "Reduce" (through adequate segregation of waste, correct management of effluents and energy, significant reduction of excesses and automatic administration of anesthetic gases), "Reuse" (through device reprocessing, reusable material and donation), "Recycle", "Rethink" (with examples such as selection of less polluting gases, selective use of containers, staff education) and "Investigate" through different models. Discussion: Several of the recognized measures could have an application in many hospital areas despite the fact that most of the available evidence refers to the operating room. The organization and education of the personnel is important in order to implement the measures found
\end{abstract}

Key words: Hospitals, medical waste disposal, recycling, equipment reuse, global warming.

\section{RESUMEN}

Introducción: La estrategia de disposición y generación de residuos influye en el cambio climático y éste, al mismo tiempo, afecta la salud de las personas, incrementando la frecuencia de enfermedades cardiovasculares, respiratorias e infecciosas entre otras. Los sistemas sanitarios son contribuyentes importantes, debido a sus altas tasas de consumo de energía, recursos y generación de desechos. Esta revisión de la literatura pretende obtener una apreciación general y actualizada de los determinantes de desechos sanitarios y las medidas implementables para disminuir la producción de residuos hospitalarios y nuestro impacto en el medio ambiente. Método: Se realizó una amplia búsqueda bibliográfica en la base de datos Medline que incluyó palabras clave relacionadas a huella de carbono, reciclaje y residuos hospitalarios. El cribado arrojó un total de 48 publicaciones. Resultados: Se

waldo.merino@ufrontera.cl

https://orcid.org/0000-0002-4956-8444 
presentan los resultados organizados en 5 puntos, a los que se les conoce como las " 5 R" y que corresponden a "Reducir" (a través de segregación adecuada de residuos, correcta gestión de efluentes, energía y disminución de excesos), "Reusar" (a través de reprocesamiento de dispositivos), "Reciclar" (transformación de residuos), "Repensar" (búsqueda de nuevas formas, innovadoras y sustentables, de las prácticas clínicas) e "Investigar" (Research, generar nuevo conocimiento). Discusión: Numerosas estrategias pueden ser implementadas para contribuir a la sustentabilidad hospitalaria y podrían tener una aplicación en muchas áreas, a pesar de que la mayoría de la evidencia disponible hace referencia al sector quirúrgico. La investigación nos ofrece herramientas para desarrollar nuevas alternativas de gestión, donde la organización y educación del personal son esenciales.

Palabras clave: Hospitales, eliminación de residuos sanitarios, reciclaje, equipo reutilizado, calentamiento global.

\section{Introducción}

propósito de esta segunda parte es, por medio de una búsqueda bibliográfica amplia, descrita en la parte I y presentada a continuación, es conocer e identificar los determinantes de desechos sanitarios y las medidas implementables; en cuanto a Repensar e Investigar; que permitan disminuir la generación de residuos de manera de alcanzar una apreciación general y actualizada de este fenómeno, teniendo en cuenta la sustentabilidad hospitalaria.

\section{Material y Métodos}

Se realizó una búsqueda que incluyó palabras claves relacionadas al cambio climático, como son huella de carbono, reciclaje y residuos hospitalarios. Para ello se realizó la siguiente búsqueda en MEDLINE: ("Hospitals"[Mesh] OR "Operating Rooms"[Mesh] OR "Surgical Procedures, Operative" [Mesh] OR "Anesthesia"[Mesh]) AND ("Medical Waste Disposal"[Mesh] OR "Recycling"[Mesh] OR "Waste Management"[Mesh] OR "Product Packaging"[Mesh]) AND ("Environmental Monitoring"[Mesh] OR "Global Warming"[Mesh] OR "Equipment Reuse"[Mesh] OR "Recycling"[Mesh] OR "Waste Management"[Mesh]) se aplicaron los filtros: 5 años, inglés, español, humanos. Ciento cuarenta y cinco resultados. Posteriormente, se realizó un cribado de los artículos en base al título y abstract disponible con los siguientes criterios de inclusión: Artículos que mencionan medidas tomadas para reducir, reutilizar o reciclar en servicios hospitalarios. Artículos que mencionan el impacto medioambiental de estas medidas o la disminución de residuos generadas. El cribado arrojó 37 documentos en total, de los cuales se realizó una lectura completa, se seleccionaron aquellos que aportaban información relevante, actualizada y/o novedosa. Posteriormente, se agregaron un total de 12 publicaciones más, a partir de las referencias de los artículos obtenidos o que eran de conocimiento de los autores. 16 de éstos fueron utilizados para ésta segunda parte, Repensar e Investigar.

\section{Resultados}

\subsection{Rethink (Repensar)}

Selección de gases y reactivos menos contaminantes: Un estudio de 2017 concluye en base a las diferencias de emisiones de $\mathrm{CO}_{2 \text { eq }}$ entre 2 hospitales de USA y uno de UK (separándolo según gases anestésicos, energía y desechos + cadena de producción) que las estrategias de reducción que incluyan evitar el uso de desfluorano como gas y la implementación de control de ventilación por demanda o "occupancy-based ventilation" (que corresponde a una ventilación inteligente) tienen el potencial para disminuir el impacto de los pabellones sin comprometer la seguridad de los pacientes. Cabe mencionar la necesidad de LCA para identificar mejor el impacto ambiental de la cadena de producción[1].

Debido al impacto ambiental negativo causado por el uso diario masivo y la eliminación de reactivos para realizar el Papanicolau, el Hospital Materno Infantil San Bartolomé de Perú modificó los componentes de la tinción de Papanicolau por reactivos menos contaminantes mostrando una sensibilidad y especificidad similar a la técnica convencional[2].

Uso selectivo de contenedores o envases. En un reporte encontraron que, entre varias drogas anestésicas, el propofol era de las más contaminantes y de las que se perdía mayor cantidad. Se pudo disminuir su pérdida de $29 \mathrm{~mL} /$ día/recipiente a 2,8 $\mathrm{mL} /$ día/recipiente simplemente cambiando las presentaciones de 50 y $100 \mathrm{~mL}$ por solo las de $20 \mathrm{ml}[3]$ lo que se explica en que es un múltiplo más pequeño.

Educación y concientización del personal. Tal como ocurría con el reciclaje mencionado anteriormente existe desconocimiento y limitaciones en el manejo de residuos. Encuestas en Canadá realizadas al personal de anestesia demostraron que existe interés en la disminución de la huella de carbono derivada de la práctica clínica en su especialidad, pero existen barreras entre las que destacan la falta de apoyo de la institución (63,5\%) e información (62,8\%)[4]. Encuestas en Etiopía realizadas a 400 trabajadores de salud de distintos estamentos organizacionales, encontraron que había poco conocimiento (53\%) y aplicación en materia de manejo de residuos $(57,7 \%)$. Características asociadas a mayor aplicación y conocimiento en manejo de residuos fueron: edad entre 35 - 44, enfermeras, matronas, personal de laboratorio, anestesiólogos y alto nivel de educación[5]. En Etiopía el 53,8\% de los trabajadores de la salud reportaron buenas prácticas de segregación de residuos y se identificaron como factores asociados a ésta el tener menos de 2 años de experiencia laboral, estar informado en precauciones estándar y la presencia de contenedores separados para los diferentes residuos[6].

Por otro lado, el equipo médico duradero, definido como un amplio grupo de dispositivos e insumos usados para el beneficio terapéutico de individuos con diferentes patologías (por ejemplo sillas de ruedas, monitores de apnea, tanques de oxígeno, etc.) es otro tema a abordar en relación con la concientización. Un estudio estadounidense basado en encuestas realizadas al personal y los pacientes encontró que: 1) Los pacientes reciben 
poca o nula información sobre el reuso o reciclaje de estos dispositivos; 2) Los hospitales implementan prácticas sustentables para reducir los desechos derivados de estos equipos cuando caducan; 3) Uno de los centros creó un programa de reuso de estos dispositivos en respuesta a ciertas necesidades locales y 4) Los "valores" de las empresas proveedoras de estos dispositivos tienen una orientación de responsabilidad medioambiental[7].

Destinación de personal específico. En el centro de salud "St. Joseph's Healthcare Hamilton (SJHH)" se realizó un plan de sustentabilidad medioambiental de 10 años (2008 - 2018) que presentó sus estrategias y resultados en un estudio, donde mencionan que uno de los puntos cruciales para el éxito en su camino hacia la sustentabilidad fue la contratación de personal destinado específicamente a coordinar el programa[8].

\subsection{Research (Investigación)}

Existen variados métodos de análisis que se han mencionado a lo largo de este artículo y que pueden ser aplicados para desarrollar nuevos estudios que contribuyan a la sustentabilidad hospitalaria, entre ellos están las auditorías, el análisis del ciclo de vida de los productos y la aplicación de encuestas en el personal y los pacientes. A continuación, mencionaremos otros puntos donde la generación de nuevo conocimiento es importante.

Iniciativas internacionales. Existen a nivel internacional iniciativas coordinadas para el avance hacia la sustentabilidad medioambiental hospitalaria, entre estas se destacan dos encontradas a través de las referencias: "Hospitales Verdes" y "Practice Greenhealth"[9],[10].

En junio de 2008 fue fundada "Practice Greenhealth", una ONG que actualmente trabaja en distintas áreas de intervención (construcción, químicos, clima, energía, desechos, comida, entre otros). Dentro de éstas cabe mencionar al proyecto "Greening the operating room (OR)". Entre las prácticas sugeridas en esta iniciativa incluyen reutilizar varios materiales a través de compañías que pudieran venderlos a un costo menor $(40 \%$ - 69\%), comprar materiales reutilizables, reformular los materiales usados en pabellón, reducir consumo de energía a través de reprogramación de los sistemas de ventilación y disminuir la producción de GEI a través de estrategias relacionadas a los gases anestésicos, entre otros, como se muestra en la Tabla 1[9]. Esta misma ONG ofrece oportunidades de intercambio de información entre los distintos miembros adscritos a sus programas donde además muchos publican los resultados de la implementación[9]. Esto último también lo realiza el proyecto de "hospitales verdes" que será mencionado más adelante[10].

En la misma línea durante el 2012, se puso en marcha la Red Global de Hospitales Verdes y Saludables (Global Green and Healthy Hospital), una iniciativa de Salud sin Daño (Health Care Without Harm) que agrupa a hospitales, sistemas de salud y organizaciones académicas vinculadas con el sector salud que buscan reducir su huella ecológica y promover la salud ambiental pública. Cuentan con más de 1.380 miembros en 72 países, contando con más de 43.000 hospitales y centros de salud. En América Latina, cuenta con 831 miembros en 14 países, que representan más de 5.600 hospitales y centros de salud. En Chile, existen más de 200 establecimientos inscritos, entre ellos hospitales, clínicas, servicios de salud, Centros de Salud Familiar, entre otros establecimientos dentro de la red asistencial. La red se

\begin{tabular}{|c|c|c|}
\hline Programas & $\begin{array}{l}\text { Ahorro } \\
\text { por OR }\end{array}$ & $\begin{array}{c}\text { Ahorro por } \\
\text { establecimiento }\end{array}$ \\
\hline $\begin{array}{l}\text { Recolección y compra } \\
\text { de dispositivos médicos } \\
\text { eprocesados }\end{array}$ & $\$ 6,970$ & $\$ 98,848$ \\
\hline $\begin{array}{l}\text { Sistemas de gestión de } \\
\text { fluidos de recipientes } \\
\text { (canister) reutilizables }\end{array}$ & $\$ 3,665$ & $\$ 27,700$ \\
\hline $\begin{array}{l}\text { Reformulación del kit del } \\
\text { oabellón }\end{array}$ & $\$ 1,786$ & $\$ 19,098$ \\
\hline $\begin{array}{l}\text { Contenedores de esterilización } \\
\text { reutilizables }\end{array}$ & $\$ 1,480$ & $\$ 19,550$ \\
\hline $\begin{array}{l}\text { Reprogramación de los } \\
\text { sistemas de ventilación para } \\
\text { disminuir el gasto cuando los } \\
\text { oabellones están en desuso } \\
\text { (HVAC setback). }\end{array}$ & $\$ 952$ & $\$ 33,604$ \\
\hline $\begin{array}{l}\text { nsumes quirúrgicos } \\
\text { reutilizables }\end{array}$ & $\$ 2,126$ & $\$ 16,750$ \\
\hline -uces LED & $\$ 146$ & $\$ 4,380$ \\
\hline $\begin{array}{l}\text { Ahorro total de las iniciativas } \\
\text { de "Greening the OR" }\end{array}$ & $\$ 53,316,828.29$ & \\
\hline
\end{tabular}

OR: Pabellón quirúrgico, del inglés operating room.

sustenta en el compromiso de sus miembros en implementar, al menos, 2 de los 10 objetivos incorporados en la Agenda Global para Hospitales Verdes y Sustentables, registrando su progreso en aquellos que se comprometieron, estableciendo sus metas año a año y compartiendo sus mejores prácticas, creando una comunidad virtual[11].

Se ha estado aplicando esta información para mejorar la sustentabilidad de distintos centros de salud, por ejemplo, el año 2018 en la Universidad de Pittsburgh, Pennsylvania, se diseñaron intervenciones para reducir la huella de carbono en histerectomía laparoscópica, en base a las recomendaciones de organizaciones sustentables de salud (entre ellas practice greenhealth y su programa "greening the OR"). Se usó el análisis del ciclo de vida de distintas actividades para estimar que intervenciones eran más útiles y poder medir los resultados. La mayor disminución en la huella de carbono fue gracias a la selección de gases anestésicos específicos y minimizando los materiales usados en cirugía. Otras fueron intervenciones relacionadas con energía, reciclaje y reuso de materiales, las que correspondieron al 10\% o menos de reducción. Todas las estrategias pudieron reducir la huella de carbono hasta en $80 \%[12]$.

Otros modelos de estudio. También dentro de la investigación se destacan algunas áreas y modelos de estudios no presentados previamente, como el que propone Romero et al., en contraste a las auditorías tradicionales. Los autores describen un modelo que considera múltiples criterios para la evaluación ambiental, específica para centros de salud, que permite conocer a cada hospital en qué estado se encuentra e identificar aquellas con menores logros, permitiendo organizarse fácilmente y definir objetivos[13]. Otro ejemplo novedoso es el de un estudio de caso realizado en un hospital de Pakistán 
el año 2018 donde se realizó un "análisis de emergía" (definida como la energía que se utiliza para transformar la materia prima en un producto) para analizar el potencial económico de segregar y reciclar distintas categorías de residuos. Estos concluyeron que la segregación adecuada y el posterior reciclaje de muchos residuos hospitalarios puede evitar los costos derivados de la extracción de recursos naturales y procesamiento de éstos, entregándoles además un valor agregado en conceptos ecológicos. Se presentó el valor económico empírico con cifras superiores a los 5.000 USD anuales al que se suma también un valor ecológico[14].

Indicadores. Para una correcta toma de decisiones por parte de cualquier establecimiento médico o institución, es necesario tener en cuenta el factor de medición y seguimiento a través de indicadores.

Los indicadores ayudan a verificar que, efectivamente, existen mejoras después de la implementación de programas o acciones tomadas. A continuación, se propone indicadores relacionados a la implementación de la metodología 5Rs como se muestra en la Tabla 2.

Para el cálculo de estos indicadores, es necesario llevar un registro periódico de los datos requeridos. Para ello se recomienda, previamente, elaborar formatos de registros y capacitar al personal que llenarán dichos formatos.

\section{Discusión}

Se reconoce al cambio climático como uno de los problemas más importantes a enfrentar en el presente siglo, por sus riesgos a la salud mundial[15]. En este contexto los sistemas sanitarios son un agente relevante en la contaminación del medioambiente y por tanto, es imperativo conocer, comprender e implementar estrategias destinadas a minimizar el impacto ecológico de la práctica clínica. Existe dentro de la literatura distintas experiencias que muestran resultados favorables en cada una de las 5 R.

Varias de las medidas reconocidas podrían tener una aplicación en cualquier área hospitalaria. Pese a esto, la mayoría de la evidencia disponible está limitada al pabellón, lo cual podría deberse a que el $20 \%$ a $30 \%$ del desecho hospitalario proviene únicamente de los pabellones[16].

La mayor parte de la evidencia y programas implementados provenían de continentes distintos al Americano, lo cual no permite conocer la realidad a nivel nacional y sudamericano. Probablemente, esta disyuntiva guarde relación a que las herramientas de búsqueda utilizadas cuentan con mayor publicación extranjera que nacional o, por otro lado, no exista gran cantidad de literatura en esta materia.

El personal tiene interés por involucrarse en las mejoras de manejo de residuos, pero existen barreras para la aplicación de estrategias, tales como: falta de liderazgos, percepción de riesgo infeccioso, falta de información, preocupación por aumento de carga laboral y resistencia al cambio[16].

Es importante establecer equipos de trabajo que involucren todos los niveles organizacionales dentro del centro hospitalario, considerando médicos, personal de enfermería, de limpieza, administrativos e incluso gobiernos locales, ya que la responsabilidad no debiera caer en una sola entidad, sino más bien ser colectiva. Como ejemplo de trabajo colaborativo se tiene la Red Global de Hospitales Verdes y Practice Greenhealth, las cuales permiten compartir herramientas, recursos y centros de datos; éstas han presentado resultados favorables, muchos de los cuales sin embargo son auto reportados o poseen un acceso restringido para los individuos no adscritos a los programas.

Para superar la resistencia al cambio es importante la educación continua específica en estas materias. Hay que considerar la sobrecarga del personal hospitalario, en relación a que se encuentran hoy trabajando al límite de su capacidad, por el aumento de la población y enfermedades crónicas. El espacio para invertir dinero y tiempo en iniciativas ecológicas se ve limitado. Con listas de espera quirúrgicas, horarios ajustados para atención de pacientes y consideraciones económicas. A menudo hay poco tiempo para contemplar el impacto del exceso y la segregación adecuada de residuos.

Sin embargo, uno de los primeros pasos para iniciar con

\begin{tabular}{|c|c|c|}
\hline Nombre & Descripción & Fórmula \\
\hline Capacitación del personal & $\begin{array}{l}\text { El indicador permite evaluar la cantidad de trabajadores } \\
\text { que se capacitan mensualmente en temas de 5Rs }\end{array}$ & Cantidad de personal capacitado / mes \\
\hline $\begin{array}{l}\text { Composición de los } \\
\text { Residuos sólidos }\end{array}$ & $\begin{array}{l}\text { El indicador muestra el \% del cual se componen los } \\
\text { residuos sólidos, según su clasificación al segregarse }\end{array}$ & $\begin{array}{l}\text { Cantidad de residuos sólidos clasificados / } \\
\text { Cantidad total }\end{array}$ \\
\hline $\begin{array}{l}\text { Generación de residuos hospitalarios } \\
\text { per cápita/mes }\end{array}$ & $\begin{array}{l}\text { Este indicador permite estimar la generación promedio } \\
\text { per cápita de residuos hospitalarios, mensualmente }\end{array}$ & Cantidad hospitalarios paciente / mes \\
\hline $\begin{array}{l}\text { Generación de residuos hospitalarios } \\
\text { per cápita por área }\end{array}$ & $\begin{array}{l}\text { Este indicador permite estimar la generación promedio } \\
\text { per cápita de residuos hospitalarios, por cada área del } \\
\text { establecimiento, mensualmente. }\end{array}$ & $\begin{array}{l}\text { Cantidad de residuos hospitalarios } \\
\text { generados / área / mes }\end{array}$ \\
\hline Emisión de efluentes & $\begin{array}{l}\text { El indicador muestra la cantidad mensual de efluentes } \\
\text { generados mensualmente }\end{array}$ & Cantidad de efluentes generados / paciente \\
\hline Emisión de gases anestésicos & $\begin{array}{l}\text { Este indicador permite evaluar la cantidad emitida total } \\
\text { de gases anestésicos mes a mes. }\end{array}$ & Cantidad de gas anestésico emitido total / mes \\
\hline Costo de los residuos hospitalarios & $\begin{array}{l}\text { Este indicador muestra los costos incurridos en la } \\
\text { disposición final de los residuos hospitalarios en cada mes }\end{array}$ & $\begin{array}{l}\text { Costo incurrido por disponer de residuos } \\
\text { hospitalarios / mes }\end{array}$ \\
\hline
\end{tabular}


este importante cambio, es la concientización del personal y la educación ambiental arraigada como parte de la cultura organizacional presente en cada trabajador. Agregado a esto, se tiene que facilitar el cambio mediante acciones contundentes y sencillas, que apoyen al personal a interiorizar lo aprendido. Un claro y recomendado ejemplo para iniciar es la correcta segregación de residuos sólidos, esto facilita el aprovechamiento y posterior valorización de los desechos y también supone un importante ahorro en costos en disposición final de residuos peligrosos. Cabe destacar que esta acción puede llevarse a cabo tanto en pabellón como en todas las áreas de un hospital.

Finalmente, se resalta que existen numerosas estrategias que pueden ser usadas para desarrollar nuevos estudios que contribuyan a la sustentabilidad hospitalaria, entre ellos están las auditorías, el análisis del ciclo de vida de los productos, la aplicación de encuestas en el personal y los pacientes, el análisis de energía y el seguimiento económico y de resultados de los proyectos que se lleven a cabo en los distintos centros. Se hace el llamado al personal médico a posicionarse como líder y participar activamente en la investigación e implementación de prácticas ecológicas.

\section{Referencias}

1. Macneill AJ, Lillywhite R, Brown CJ. Articles The impact of surgery on global climate : a carbon footprinting study of operating theatres in three health systems. Lancet Planet Heal [Internet]. 2017;1(9):e381-8. Disponible en: https://doi.org/10.1016/S25425196(17)30162-6. [consultado el 27 de enero de 2021].

2. Moya-Salazar J, Rojas-Zumaran V. Eco-Pap: The Ecological Modification of the Papanicolaou Stain for Sustainable Cervical Cancer Diagnosis. Acta Cytol. 2019;63(1):35-43. https://doi. org/10.1159/000493113 PMID:30352442

3. Mankes RF. Propofol wastage in anesthesia. Anesth Analg. 2012 May;114(5):1091-2. https://doi.org/10.1213/ ANE.0b013e31824ea491 PMID:22415537

4. Petre MA, Bahrey L, Levine M, van Rensburg A, Crawford M, Matava $C$. A national survey on attitudes and barriers on recycling and environmental sustainability efforts among Canadian anesthesiologists: an opportunity for knowledge translation. Can J Anesth. 2019;66(3):272-86. Disponible en: https://doi. org/10.1007/s12630-018-01273-9.

5. Doylo T, Alemayehu T, Baraki N. Knowledge and Practice of Health Workers about Healthcare Waste Management in Public
Health Facilities in Eastern Ethiopia. J Community Health [Internet]. 2019;44(2):284-91. Disponible en: https://doi.org/10.1007/ s10900-018-0584-z.

6. Sahiledengle B. Self-reported healthcare waste segregation practice and its correlate among healthcare workers in hospitals of Southeast Ethiopia. BMC Health Serv Res. 2019 Aug;19(1):591. https://doi.org/10.1186/s12913-019-4439-9 PMID:31438959

7. Ordway A, Pitonyak JS, Johnson KL. Durable medical equipment reuse and recycling: uncovering hidden opportunities for reducing medical waste. Disabil Rehabil Assist Technol [Internet]. 2020;15(1):21-8. Disponible en: https://doi.org/10.1080/174831 07.2018.1508516. [consultado el 27 de enero de 2021].

8. Langstaff K, Brzozowski V. Managing environmental sustainability in a healthcare setting. Healthc Manage Forum. 2017 Mar;30(2):84-8. https://doi.org/10.1177/0840470416675178 PMID:28929883

9. Practice Greenhealth. Greening the OR / Practice Greenhealth [Internet]. Disponible en: https://practicegreenhealth.org/topics/ greening-operating-room/greening-or [consultado el 27 de enero de 2021].

10. Greenhealth P. 2019 Sustainability Benchmark Data Data tables Introduction and methods. 2019.

11. Red Global de Hospitales Verdes y Saludables [Internet]. Disponible en: https://www.hospitalesporlasaludambiental.net/ [consultado el 27 de enero de 2021].

12. Thiel CL, Woods NC, Bilec MM. Strategies to Reduce Greenhouse Gas Emissions from Laparoscopic Surgery. Am J Public Health. 2018 Apr;108 S2:S158-64. https://doi.org/10.2105/ AJPH.2018.304397 PMID:29698098

13. Carnero MC. Environmental assessment in health care organizations. 7th Int Multi-Conference Complexity, Informatics Cybern IMCIC 2016 7th Int Conf Soc Inf Technol ICSIT 2016 - Proc. 2016;1(Carnero 2015):123-8.

14. Ali M, Geng Y. Accounting embodied economic potential of healthcare waste recycling-a case study from Pakistan. Environ Monit Assess. 2018 Oct;190(11):678. https://doi.org/10.1007/ s10661-018-7063-y PMID:30368602

15. Ramanathan $V$, Haines $A$. Healthcare professionals must lead on climate change. BMJ. 2016 Oct;355(October):i5245. https://doi. org/10.1136/bmj.i5245 PMID:27702748

16. Wyssusek KH, Keys MT, van Zundert AA. Operating room greening initiatives - the old, the new, and the way forward: A narrative review. Waste Manag Res. 2019 Jan;37(1):3-19. https://doi. org/10.1177/0734242X18793937 PMID:30132405 